ArtefaCToS. Revista de estudios de la ciencia y la tecnología

eISSN: $1989-3612$

Vol. 9, No. 1 (2020), 2. ${ }^{\text {a }}$ Época, 61-76

DOI: https://dx.doi.org/10.14201/art2020916176

\title{
La dimensión social de la Tecnología: El control de armas químicas y biológicas
}

\section{The Social Dimension of Technology: The Control of Chemical and Biological Weapons}

\author{
Brian BALMER \\ University College London, Reino Unido \\ b.balmer@ucl.ac.uk
}

Recibido: 26/01/2020. Revisado: 08/02/2020. Aceptado: 16/02/2020

\section{Resumen}

Este artículo aborda cuestiones conceptuales que surgen de los intentos de los gobiernos y la sociedad civil por controlar la diseminación de armas químicas y biológicas. La primera parte versa sobre aspectos clave del control internacional de estas armas, enfocada hacia los principales tratados que las proscriben. Luego, se destacan dilemas prácticos y conceptuales clave para practicar estos controles. Son los problemas de definición, del "doble uso", de cómo distinguir la investigación defensiva de la ofensiva, de la verificación y de la dificultad de analizar la guerra química y biológica desde una perspectiva cultural. También esta segunda parte trata investigaciones del autor sobre la Historia de la guerra química y biológica, y ańade reflexiones sobre cómo este trabajo podría informar algunos de los asuntos prácticos contemporáneos en el control de esta guerra.

La contribución que los filósofos pueden hacer (y hacen) para el control de las armas químicas y biológicas se da en el campo ético; otros debates de la Filosofía de la Ciencia, sobre términos como conocimiento tácito, Ontología, infradeterminación y Filosofía feminista de la Ciencia tienen el potencial de arrojar luz sobre los dilemas que afronta la comunidad del control de armas.

Palabras clave: conceptos; diseminación; guerra; desarme; ética; comunidad. 


\begin{abstract}
This paper deals with a number of conceptual issues that arise from attempts by governments and civil society to control the spread of chemical and biological weapons. The first part outlines key aspects of the international control of chemical and biological weapons, focusing on the main treaties that outlaw these weapons. It then highlights some of the key practical and conceptual dilemmas in implementing these controls. These are the problems of definition, 'dual-use', how distinguishing defensive from offensive research, verification, and difficulty of analyzing chemical and biological warfare from a cultural perspective. Also this second part contains a discussion of some of the author's own research on the history of chemical and biological warfare, and adds some reflections on how this work might inform some of the contemporary practical issues in the control of chemical and biological warfare.

The most obvious contribution that philosophers can (and do) make to the control of chemical and biological weapons is in the field of ethics; the author argues that other debates from philosophy of science, over terms such as tacit knowledge, ontology, underdetermination and feminist philosophy of science, have the potential to shed further light on the dilemmas facing the arms control community.
\end{abstract}

Keywords: concepts; spread; warfare; disarmament; ethics; community.

\title{
1. Introducción
}

Muy poco después de los atentados terroristas del 11 de septiembre de 2001 a las Torres Gemelas, en Nueva York y al Pentágono, en Arlington (Virginia), en algún momento alrededor del 18 de septiembre, en el área de Princeton (Nueva Jersey) se envió la primera de las dos tandas de cartas a varias sucursales de medios de comunicación, una [sucursal] en Florida y las demás en Nueva York. Las cartas advertían que contenían ántrax y, de hecho, se les habían echado esporas letales de ántrax, aunque en un principio se descartó como un engaño (cfr. Cole, 2003; Guillemin, 2011). El 3 de octubre se diagnosticó a la primera víctima y falleció una semana después. Mientras se diagnosticó a los demás, se confirmó que las cartas eran la fuente de las infecciones de ántrax. Después, en algún momento alrededor del 8 de octubre, se envió un ulterior conjunto de cartas, incluyendo a dos senadores demócratas en Washington D.C. Finalmente, se produjeron doce muertes por ántrax. La teoría principal del FBI, en su subsiguiente investigación de los ataques, era que fueron perpetrados por un delincuente nacional, quizá un empleado descontento del programa estadounidense de defensa de la guerra biológica ( $B W$, biological warfare). Algún tiempo después, el FBI mencionó a Bruce Ivins, un científico senior en el Army Medical Research Institute of Infectious Diseases [Instituto de Investigación Médica de Enfermedades Infecciosas del Ejército],

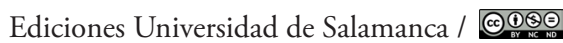

ArtefaCToS, Vol. 9, No. 1 (2020), 2. á́poca, 61-76 
en Fort Detrick, como su principal sospechoso. Ivins se suicidó en julio de 2008, por lo que el FBI no pudo llevar un caso a los tribunales.

Algunos años antes de los ataques con ántrax, una secta japonesa llamada Aum Shinrikyo lanzó ataques terroristas, no con armas biológicas vivas que provocan enfermedades, sino con armas químicas inertes altamente tóxicas. El primer ataque en Matsumoto, una ciudad turística e industrial a unas cien millas al noroeste de Tokyo, tuvo lugar el 27 de junio de 1994. Los miembros de la secta emplearon un camión diseñado para propagar el gas sarín (el sarín es un agente químico que ataca el sistema nervioso rápidamente). Estacionaron frente a un supermercado y liberaron el sarín, que se dispersó por los apartamentos cercanos y mató a siete personas y causó daños a alrededor de 250. Esto fue despachado por las autoridades japonesas como un accidente de un aficionado a la Quími$\mathrm{ca}$, un acontecimiento trágico producido después de que alguien hubiera estado jugando con pesticidas. El segundo ataque tuvo lugar el 20 de marzo de 1995, y esta vez la secta seleccionó como objetivo el sistema subterráneo de Tokio. Aquí el sarín simplemente se colocó en bolsas de plástico selladas que, después, los miembros de la secta perforaron antes de escapar rápidamente de las bolsas mortíferas. Entonces el sarín se dispersó gradualmente, provocando el caos y matando a unas doce personas, dejando a 17 en estado crítico, a otras 37 con síntomas graves (dificultad respiratoria, vómitos, jaqueca, espasmos musculares, problemas gastrointestinales) y a alrededor de otras 1.000 personas con síntomas leves (cfr. Tucker, 2007).

Ambos atentados ponen de relieve la realidad actual de la amenaza de las armas químicas y biológicas. Además de estos ejemplos, es aleccionador recordar que, en el siglo XX, fueron los Estados nación los que mostraron mayor interés en estas armas, con muchos de ellos llevando a cabo esfuerzos altamente secretos y patrocinados por el Estado para aplicar la Ciencia y la Tecnología a la creación de agentes químicos y biológicos incluso más mortíferos (cfr. Wheelis, Rózsa y Dando, 2006; Coleman, 2005; y Harris y Paxman, 1982). Sin embargo, tampoco deberíamos perder de vista los muchos esfuerzos que se han hecho para controlar la amenaza de estos agentes y esto será el principal centro de este trabajo.

El artículo se estructura en tres partes. La primera parte simplemente esbozará los aspectos clave en el control internacional de armas químicas y biológicas, centrándose en los principales tratados que prohíben estas armas. Es necesariamente descriptiva. Después de esta descripción, destacaré algunos de los dilemas clave, prácticos y conceptuales, en la puesta en práctica de estos controles. Estos son los problemas de definición, el problema de "uso dual" (dual-use), el problema de distinguir la investigación defensiva de la investigación ofensiva y el problema de la verificación. Finalmente, quiero dar a conocer un poco de mi investigación en colaboración, un estudio de caso histórico de uso dual y del descubrimiento de los denominados agentes nerviosos de serie V durante la Guerra Fría. Termino con algunas breves reflexiones sobre cómo este estudio de caso podría proporcio- 
nar información para algunos de los problemas prácticos contemporáneos en el control de la guerra química y biológica.

\section{Tratados de control de armas}

Los tratados para el control de armas (arms control treaties) no son la única forma de controlar la guerra química y biológica. El desarme total, la coacción y las sanciones son ejemplos de otros medios. Dando seńala que todo tratado de control de armas: (1) Establece un "régimen" para gobernar una Tecnología armamentística (esto consiste en normas, leyes, organizaciones etc., en otras palabras, el tratado no es solo una hoja de papel); (2) los beneficios de firmar deben ser mayores que los costes; (3) siempre hay una necesidad de equilibrar (balance) una variedad de intereses (en particular, cuando la guerra química y biológica tiene fuertes coincidencias con la investigación civil en áreas como la investigación agrícola y farmacéutica; (4) a este respecto, los tratados no solo versan sobre seguridad internacional —afectan a la industria y a la comunidad científica-; y (5) hay diferentes funciones de verificación (un tratado ideal atraparía a los infractores, pero si esto se ve como demasiado idealista, todavía puede haber objetivos más modestos, como establecer un marco para expresar preocupaciones, poner obstáculos a los potenciales infractores [perpetrators], etc.) (cfr. Dando, 2002).

\section{Control de armas químicas y biológicas}

El primer uso a gran escala de armas químicas tuvo lugar durante la Primera Guerra Mundial, aunque hay muchos ejemplos de un uso más limitado de armas químicas y biológicas que se remontan a la Antigüedad (cfr. Mayor, 2003). Después del uso de sustancias químicas como la clorina, el fosgeno y el gas mostaza (chlorine, phosgene and mustard gas) durante la Primera Guerra Mundial, el período de entreguerras asistió a nuevos intentos de prohibir las armas químicas. Estos esfuerzos culminaron, a través de negociaciones en la Sociedad de Naciones, en el Protocolo de Ginebra de 1925, un tratado que establecía la prohibición tanto de la guerra química ("uso en la guerra de gases asfixiantes, venenosos o de otro tipo, y de todos los líquidos, materiales o dispositivos análogos”) como de la guerra "bacteriológica". El tratado estuvo lejos de ser ideal. Lo que se consideraba como "uso en la guerra" quedaba sin especificar. De forma significativa, algunas naciones, en particular Estados Unidos, no ratificaron el tratado, mientras que muchas otras tuvieron reservas. En consecuencia, el Protocolo fue efectivamente un acuerdo de "no-primer-uso" (not-first-use) de estas armas más que una prohibición rotunda.

A finales de los años 60, el desarme químico y biológico también había vuelto a la agenda internacional. Mientras que el tema nunca se había abandonado completamente, el impulso para el control de armas se incrementó mediante 
grandes avances en la limitación de armas nucleares; a saber, el Limited Test Ban Treaty, de 1963, [Tratado de limitación de pruebas] y, después, en 1968, el Nuclear Non-Proliferation Treaty [Tratado de no-proliferación nuclear] (cfr. Walker, 2012). A finales de la década de 1960, una serie de negociaciones internacionales intentaron, primero, forjar una convención que prohibiese tanto las armas químicas como las biológicas. Por razones que solo ahora comienzan a apreciar los historiadores y que van más lejos que el alcance de este artículo, se separaron las dos categorías de armas. El resultado fue la Biological Weapons Convention (BWC) [Convención sobre Armas Biológicas], en 1972 y solo mucho después, en 1997, la Chemical Weapons Convention (CWC) [Convención sobre Armas Químicas].

La BWC estuvo lista para firmar el 10 de abril de 1972 y entró en vigor el 26 de marzo de $1975^{1}$. En la actualidad, el tratado cuenta con 163 estados firmantes (incluidos el Reino Unido y España). Se erige como el primer tratado que prohíbe una clase completa de armas. También es un tratado extremadamente corto, con unas pocas páginas de extensión. El núcleo del tratado es el artículo primero, donde cada Estado participante que haya firmado el tratado "se compromete a no desarrollar, producir, almacenar o, de otra forma, adquirir o retener, nunca y bajo ninguna circunstancia: (1) Agentes microbianos u otros agentes biológicos, o toxinas, cualesquiera que sean su origen o método de producción, de tipos o en cantidades que no estén justificados para fines profilácticos, de protección u otros fines pacíficos; (2) las armas, equipos o vectores diseñados para emplear esos agentes o toxinas con fines hostiles o en conflictos armados".

Aunque la negociación de la CWC comenzó paralelamente a las negociaciones de la BWC en agosto de 1968, el tratado se acordó y, después, quedó listo para firmar ya en enero de 1993. Las negociaciones pasaron por muchos cambios en el escenario geopolítico, incluyendo la Guerra de Vietnam, la distensión e intensificación de la Guerra Fría - y, más adelante, su final — y, después, la Guerra del Golfo. Mientras que esto supuso negociaciones prolongadas, el tratado finalmente entró en vigor 180 días después de su ratificación por el sexagésimo quinto país firmante (Hungría) y entró en vigor el 29 de abril de $1997^{2}$.

El tratado es un documento mucho más extenso que el BWC, con 24 artículos y tres anexos. Sus principales disposiciones son las siguientes:

- Cada Estado participante se compromete a no adquirir, almacenar o conservar armas químicas o a transferir, directa o indirectamente, armas químicas a nadie, nunca y bajo ninguna circunstancia.

- No utilizar armas químicas o llevar a cabo preparativos militares para utilizarlas.

\footnotetext{
${ }^{1}$ Cuando entra en vigor un tratado sus disposiciones se convierten en legalmente vinculantes para los Estados-parte en la convención.

${ }^{2}$ Véase [OPCW, Organization for the Prohibition of Chemical Weapons, The Implementing Body for the Chemical Weapons Convention (CWC),] www.opcw.org [acceso 7.1.2020].
} 
- No ayudar, alentar o inducir en modo alguno a nadie que realice cualquier actividad prohibida por el tratado.

- Desarme: Compromiso de destruir, en el plazo de 10 años, las armas químicas que posean los firmantes, las instalaciones de producción y las armas abandonadas en el territorio de otro Estado participante.

Como he mencionado, mientras que los tratados son medios cruciales para prevenir el uso incorrecto de las armas químicas y biológicas, solo constituyen un aspecto de lo que se ha denominado "red de disuasión" (web of deterrance) (cfr. BMA, 1999). Otros medios para controlar estas armas incluyen el seguimiento y conocimiento de la exportación, la investigación en medidas defensivas y de protección, las respuestas nacionales e internacionales a la adquisición y/o el uso de armas químicas y biológicas (por ejemplo, las inspecciones de la UNSCOM [Comisión Especial de la ONU] que siguieron a la primera guerra de Irak no se llevaron a cabo bajo la BWC) y la introducción de códigos de conducta para los científicos. En lugar de describir estas medidas en detalle, quiero pasar a debatir los problemas del control de armas químicas y biológicas. Creo que esto plantea algunos problemas conceptuales más a los que creo que pueden hacer frente los académicos de Historia de la Ciencia, Filosofía de la Ciencia y Estudios sociales de la Ciencia.

\section{Problemas del control de armas químicas y biológicas}

\subsection{Definiciones}

¿Qué se entiende por agente biológico? ¿En qué medida tiene que ser tóxica una sustancia química para que se considere un agente químico? Las armas biológicas se definen, de manera convencional, como aquellas que emplean organismos vivos (living organisms) para causar enfermedades en humanos, otros animales o cultivos (cfr. Dando, 1994). Las armas químicas hacen referencia, por lo general, a agentes químicos inertes cuya forma principal de causar daño es su toxicidad, diferenciándose así de otras armas como los artefactos incendiarios. Como tal, pueden crear ampollas, provocar asfixia, envenenar la sangre e interferir en el sistema nervioso para incapacitar o matar (cfr. Kenyon, 2000).

Estas definiciones todavía plantean problemas para el control de armas. Destaca que los dos tratados no dan una lista de sustancias prohibidas. Esto se debe a varias razones. Como se trata aquí, muchos agentes químicos y biológicos son de "uso dual" (dual-use); en otras palabras, pueden emplearse para fines benignos o malignos. Una lista también dejaría el tratado abierto al punto débil según el cual se podría pensar que están fuera del alcance de los tratados nuevos agentes futuros, como agentes químicos recién sintetizados u organismos genéticamente modificados. 
Ambos tratados sortean estos problemas empleando el denominado Criterio de la Finalidad General (General Purpose Criterion). Esto no regula los agentes específicos, sino que regula las intenciones. Así, por ejemplo, la BWC declara que prohíbe los "agentes microbianos u otros agentes biológicos, o toxinas, cualesquiera que sean su origen o método de producción, de tipos o en cantidades que no estén justificados para fines profilácticos, de protección u otros fines pacíficos" (cursivas añadidas). La posesión de algún agente biológico podría justificarse si, por ejemplo, se demostrara que se iba a utilizar para hacer vacunas; pero podrían prohibirse si se probara que el agente estaba pensado para una bomba ${ }^{3}$. Mientras que el Criterio de la Finalidad General evita los problemas de hacer listas, también tiene la desventaja de hacer muy difíciles el cumplimiento (compliance) y la verificación (verification), en tanto que ahora se necesita probar (prove) la intención.

\subsection{El problema del uso dual (dual-use)}

Como se ha mencionado, un problema central para la puesta en práctica de controles sobre la guerra química y biológica es el denominado dilema del uso dual. La historia de ambos tipos de armas descansa en la investigación civil —la investigación sobre la salud y la industria química- $-\mathrm{y}$ es posible concebir usos pacíficos y dañinos para muchos agentes. Así, por ejemplo, el tiodiglicol químico es precursor clave del gas mostaza, pero también se utiliza para producir tinta para bolígrafos. Otro ejemplo es la ricina, que se extrae de las semillas del ricino. Se cultiva para el comercio de aceite de ricino, que más allá de los usos medicinales tiene muchas aplicaciones industriales (por ejemplo, para producir pintura de resina, barnices, polímeros sintéticos de nailon, cosméticos e insecticidas) (cfr. Tucker, 1994). También es altamente tóxica y ha sido estudiada durante el siglo XX como arma potencial en los programas de investigación estadounidenses, canadienses, británicos, franceses y japoneses sobre armas biológicas.

Un problema relacionado con este se presenta cuando los científicos en escenarios civiles emprenden una investigación con fines pacíficos, pero descubren por casualidad algo con un uso potencialmente maligno. En 2001, por ejemplo, unos científicos estaban trabajando en Australia sobre la viruela en ratones con el objetivo de controlar las plagas en emplazamientos domésticos y agrícolas. Se suponía que el virus genéticamente modificado iba a actuar "trucando" (tricking) el sistema inmune del ratón, para reaccionar contra sus propios óvulos, esterilizan-

\footnotetext{
${ }^{3}$ La CWC también enumera 14 familias de sustancias químicas y 29 sustancias químicas individuales que incluyeron para su control y que agruparon en tres "listas" (schedules) (siendo una de ellas la más tóxica), con la obligación de declararlas y con restricciones sobre las cantidades poseídas y producidas anualmente. A pesar de esta lista, los Estados tienen todavía la obligación de controlar todas las sustancias químicas bajo el Criterio de Finalidad General (General Purpose Criterion), no solo aquellas que figuran en la lista.
} 
do efectivamente al ratón. De hecho, los científicos crearon accidentalmente una versión altamente letal del virus, que exterminó toda su población experimental de ratones. A su vez, esto planteó problemas de si podría o no aplicarse este nuevo conocimiento, por ejemplo, al virus relacionado de la viruela en humanos (cfr. Selgelid, 2007).

Subyacen a este problema práctico algunos dilemas conceptuales más sobre qué hace a una sustancia peligrosa y sobre si el "uso dual" es una propiedad inherente de algún agente particular. Volveré sobre este tema más tarde con un estudio de caso.

\subsection{El problema de la superposición defensa/ataque}

También hay una distinción borrosa entre guerra biológica y química ofensiva y defensiva (cfr. Strauss y King, 1986). Los países que han firmado la BWC y la CWC se han comprometido a abandonar el trabajo ofensivo en armas químicas y biológicas (CBW, Chemical and Biological Weapons), pero se les permite continuar la investigación sobre medidas defensivas. Ahora bien, mientras que mejorar una máscara de gas, por ejemplo, es claramente una medida defensiva, hay ejemplos de medidas "defensivas" mucho más ambiguas. En 2002, tres periodistas estadounidenses revelaron proyectos altamente secretos emprendidos en Estados Unidos: fabricar una bomba biológica basada en un diseño soviético; construir una fábrica de armas biológicas en el desierto de Nevada utilizando únicamente material comprado a fuentes legales, tales como tiendas de hardware; e investigar sobre una cepa de ántrax resistente a la vacuna. Cuanto estos hechos se hicieron públicos, fueron justificados por las autoridades como investigación defensiva; pero, al mismo tiempo, surge la pregunta de dónde podría trazarse la línea y de cómo los Estados Unidos habrían reaccionado si otros países hubieran llevado a cabo la misma investigación.

\subsection{El problema de la verificación}

¿Cómo sabríamos si alguien ha violado el tratado y utilizado armas químicas o biológicas? Investigar los presuntos ataques con armas químicas y biológicas no es sencillo, en particular con armas biológicas, donde puede ser difícil o imposible diferenciar entre un brote natural o un brote deliberado de una enfermedad (cfr. Clunan, Lavoy y Martin, 2008). Un ejemplo de esto ocurrió en la antigua Unión Soviética en 1979. A principios de los años ochenta empezaron a aparecer informes en el oeste sobre un brote inusual de ántrax en 1979, en Sverdlovsk, a unas 900 millas al este de Moscú (cfr. Guillemin, 1999). En una ciudad de 1.200.000 habitantes, se informó de que cientos de residentes habían muerto durante un período de un mes. Estados Unidos planteó la cuestión en la reunión internacional para la revisión de la BWC de 1980, exigiendo una explicación. 
Los soviéticos afirmaron que el brote se había producido por las personas que comieron carne contaminada que se había vendido en el mercado negro. Estados Unidos nunca hizo una acusación formal contra la URSS por violar el artículo primero de la Biological Weapons Convention. En lugar de eso, prefirieron abordar el asunto a través de un artículo de la Convención con menor carga política, el artículo 5, según el cual los Estados participantes acordaban hacer consultas bilaterales y multilaterales para solucionar cualquier problema relacionado con la aplicación de la BWC.

Los documentos de los archivos del Reino Unido que acababan de salir a la luz muestran lo difícil que era establecer de un modo definitivo la causa del brote, a pesar de que la inteligencia estadounidense sabía que una instalación militar secreta, que se pensaba que era una instalación para la investigación sobre armas biológicas, se localizaba en Sverdlovsk ${ }^{4}$. Incluso si se hubiera rastreado el origen del brote en esa instalación, no habría habido ninguna manera de establecer si la actividad que tenía lugar allí había sido investigación defensiva u ofensiva.

\section{Un estudio de caso: El descubrimiento de los gases nerviosos de serie V}

He esbozado, en líneas generales, los principales problemas a los que se enfrenta el control de armas químicas y biológicas. Como se ha mencionado, aunque son problemas prácticos para los que afrontan la actuación pública (policy-makers), también plantean problemas conceptuales clave sobre la índole de estas armas y sobre cómo podemos saber si se han utilizado con fines malignos. Quiero volver ahora sobre un estudio de caso más detallado de mi propia investigación, llevada a cabo en colaboración con la Dra. Caitriona McLeish de la Universidad de Sussex, en el Reino Unido, para ilustrar cómo los estudios históricos, sociales y filosóficos de la Ciencia y la Tecnología pueden proporcionar alguna luz sobre estos problemas más conceptuales. El estudio del caso es nuestro trabajo de archivo sobre la historia de los gases nerviosos en el Reino Unido durante la Guerra Fría (cfr. McLeish y Balmer, 2012).

Los gases nerviosos son compuestos organofosforados altamente tóxicos (compuestos orgánicos que contienen fósforo). Los agentes $\mathrm{G}$ y $\mathrm{V}$ son una subcategoría de esa clase de agentes de la guerra química, designados como gases nerviosos, porque interfieren en la transmisión de señales nerviosas a través de los contactos (sinapsis) entre las células nerviosas (neuronas) o entre una neurona y un órgano que se activa por un impulso nervioso (un órgano efector) como, por ejemplo, un músculo. El agente nervioso interfiere con la seńal química (acetilcolina), mediante el bloqueo de la encima que frena esta señal química, una vez que ha cumplido su función prevista (colinesterasa). En efecto, el nervio continúa emitiendo señales y señales sin parar. Los síntomas de la intoxicación por agentes

\footnotetext{
${ }^{4}$ Cfr. The National Archives (TNA), Kew, FCO 66/1520 y FCO 66/1521.
} 
nerviosos incluyen convulsiones importantes, seguidas de una parálisis flácida en la que los músculos respiratorios dejan de funcionar, lo que lleva a la muerte por asfixia en cuestión de minutos.

Históricamente, los gases nerviosos pasaron por dos generaciones. La primera generación, conocida como los agentes G, se descubrió en Alemania durante el desarrollo de la Segunda Guerra Mundial; la segunda generación (los agentes V), se descubrieron a comienzos de los ańos 50, en el Reino Unido, en el escenario de la Guerra Fría. En ambos casos, los descubrimientos se hicieron inicialmente en el sector civil, durante el curso de la investigación civil sobre pesticidas y se transfirieron a la investigación militar. Esto hace del estudio de caso un interesante estudio de uso dual.

Los agentes $\mathrm{G}$ se descubrieron en el desarrollo de la investigación sobre pesticidas, unos ańos antes del estallido de la guerra; en un principio fue por el Dr. Gerhard Schrader, que trabajaba para el gigante químico IG Farben (cfr. Tucker, 2007). Schrader trabajó en los pesticidas que tenían por objetivo los gorgojos en el grano almacenado y las pulgas en los barcos y en escenarios domésticos. Schrader trató de sintetizar y contrastar (test) diferentes compuestos, utilizando finalmente compuestos organofosforados que se ha probado (prove) que son altamente tóxicos para los humanos, haciéndolos, por tanto, inadecuados como pesticidas.

Sin embargo, esta misma propiedad implicó que eran armas potenciales. Una ordenanza oficial del Reich de 1935 exigía que la Oficina de Guerra fuera informada de todos los nuevos descubrimientos y patentes de importancia militar potencial, la cual podría declararlos secretos. IG Farben transmitió los resultados del nuevo compuesto a la Oficina de Guerra, que a su vez transmitió la información al Laboratorio de Protección contra el Gas del ejército alemán en Spandau, Berlín. Allí se desarrolló como el primero de una serie de agentes $\mathrm{G}$ (denominado tabun) y se devolvió a IG Farben para su producción.

Finalmente, la guerra química no se empleó durante la Segunda Guerra Mundial. En el Reino Unido, durante un corto período de tiempo que siguió al fin de la Segunda Guerra Mundial, se le dio alta prioridad, por igual, a la investigación sobre armas químicas, biológicas y atómicas en la política de defensa del Reino Unido (cfr. Balmer, 2001). Un factor principal que contribuyó al nuevo entusiasmo por las armas químicas fue el descubrimiento de los gases nerviosos alemanes. Estos solo fueron descubiertos por los aliados cuando encontraron municiones alemanas rellenas con el agente tabun en las fases finales de la guerra. Pero señalaron toda una clase nueva de agentes altamente tóxicos y de acción rápida para la guerra química, que podrían ser investigados por científicos de la Chemical Defence Experimental Station [Estación Experimental de Defensa Química] del Reino Unido en Porton Down en Wiltshire. Fue re-denominada en 1948 como el Chemical Defence Experimental Establishment, CDEE [Establecimiento Experimental de Defensa Química]. 
A comienzos de la década de 1950 hubo sugerencias de los asesores científicos del CDEE acerca de que había alcanzado su límite el trabajo sobre los agentes G. En febrero de 1952, tuvo lugar un debate denominado "trabajo ulterior en los agentes G" entre los miembros de la Chemical Defence Advisory Board, CDAB [Junta Asesora de la Defensa Química], un comité asesor técnico de Porton Down, y concluyó que "era probable que sólo surgieran pocas cosas de valor de la investigación sobre cambios ulteriores en las agrupaciones de gases nerviosos conocidos".

Incluso ya en noviembre de 1950, los miembros de la Junta discutieron cómo proceder en la búsqueda de más agentes tóxicos. Para el número limitado de científicos de Porton, la aparente dificultad descansaba en predecir la toxicidad de las estructuras químicas. Esto había dado lugar a algo así como un enfoque de ensayo y error (trial-and-error approach), como se explica en esta cita extraída de una carta escrita por el Director de Investigación Química del Ministerio de Abastecimiento quien formó parte del CDAB: "Los métodos de enfoque en este momento son principalmente empíricos y, en pocas palabras, para un personal de una proporción mayor de lo que podemos conseguir, de modo que solo los bordes [del asunto] se pueden alcanzar. En consecuencia, el éxito depende en gran medida de un golpe de suerte. Este estado de cosas se considera por todos los interesados como peligroso e insatisfactorio"s.

Esto proporcionó un incentivo para que los asesores del CDEE consideraran otros dos medios: la revisión de la literatura y pedir ayuda a la industria.

El CDEE ya tenía una relación histórica con los representantes de la industria química. Muchas de las empresas que el gobierno había llamado a filas para producir gases venenosos durante la Primera Guerra Mundial se habían fusionado en Imperial Chemical Industry (ICI) [Industrias Químicas Imperiales]. Después, fue al ICI a donde acudió el gobierno en 1937, para construir y poner en funcionamiento fábricas que harían y rellenarían municiones con agentes de la guerra química. Este trabajo también se superponía a la investigación civil. El historiador del ICI William Reader cita un memorándum de 1945 donde se señala, en términos velados, que el trabajo del ICI en herbicidas "se fusionó en gran medida con la investigación en ciertos proyectos militares del más alto grado de secreto" (Reader, 1975, 458).

El nuevo impulso para solicitar la ayuda de la industria química se llevó a cabo desde el Ministerio de Abastecimiento. En enero de 1951, se escribió una carta a la asociación comercial de la industria química, la Association of British Chemical Manufactures, ABCM [Asociación Británica de Fábricas de Productos Químicos]. En ella, se solicitaba información sobre cualquier compuesto altamente tóxico descubierto recientemente. En lugar de provocar una avalancha de

5 TNA WO 188/2716 Carta para James Davidson Pratt de la Association of British Chemical Manufacturers de AE Childs, DCDRD, Ministerio de Abastecimiento, (4 de enero de 1951). 
información, los esfuerzos de búsqueda iniciales de Porton parecieron encontrar una barrera significativa en la necesidad de proteger los secretos comerciales.

Como forma de remediarlo, antes de que se enviara una segunda ronda de cartas en julio de 1953, se redactó un sistema de códigos-C (comerciales). A los nuevos compuestos enviados ahora al Ministerio de Abastecimiento se les daba ahora un número $\mathrm{C}$, de modo que, en lugar de revelar el nombre de la empresa originaria, se transmitió, en cambio, este número al CDEE con algunos detalles ulteriores sobre su composición y propiedades. Las cartas se enviaron directamente a empresas químicas y médicas (19 se nombraron en una lista) y, a través de la Asociación Británica de Fábricas de Productos Químicos, que contactó con 25 compañías. La carta solicitaba que la Asociación divulgara entre sus miembros la petición de información del Ministerio sobre compuestos tóxicos. Las razones eran que los avances en Química y en campos afines implicaban que, ocasionalmente, podían salir a la luz "tipos nuevos e inesperados (unexpected) de materiales tóxicos". Para planificar medidas de protección contra potenciales amenazas de armas químicas, continuaba:

"Debemos, por tanto, apreciar la cooperación de las organizaciones industriales y de otras organizaciones de investigación al proporcionarnos datos sobre la síntesis y las propiedades de cualquier nuevo compuesto que preparéis (o que extraigáis de productos naturales) y que muestren alta toxicidad o una toxicidad asociada con nuevas estructuras moleculares o una toxicidad de un nuevo tipo".

Esta vez el llamamiento provocó alguna respuesta; en particular, una carta del Ministerio de Abastecimiento al Director de Investigación del ICI en julio de 1953 expresa un agradecimiento velado: "En el pasado, hemos estado muy agradecidos por la cooperación del ICI y esperamos que podamos contar con él en el futuro. Para su información, el último elemento (item) recibido de usted está ahora a buen recaudo dentro de la zona alambrada y está recibiendo mucha atención"

Como veremos, en la investigación sobre nuevos compuestos tóxicos en este momento, posiblemente el único punto que provoca "mucha atención" era un nuevo pesticida organofosforado llamado amiton.

Tras el fin de la Segunda Guerra Mundial, las industrias pesticidas e insecticidas se habían extendido rápidamente por toda Europa, trabajando muchas empresas sobre compuestos organofosforados. Entre 1952 y 1953, al menos tres empresas encontraron un grupo de compuestos organofosforados que tenían una actividad insecticida muy potente; en especial, frente a los ácaros. Uno de estos compuestos, el amiton (que, más tarde, recibiría el nombre en clave Agente VG por parte del Cuerpo Químico del Ejército estadounidense) fue un aspirante a

${ }^{6}$ TNA, WO 188/2721. Carta de J. McCaulay a R. M. Winter, Director de investigación, Messrs ICI S.A., Nobel House, Buckingham Gate (15 de Julio de 1953). 
agente acaricida. Fue descubierto por Ghosh y Newman, que trabajaban para Plant Protection LImited (PPL), una filial de dos compañías ICI, y Cooper, MacDougall y Robertson (CMR).

Probablemente, Ghosh sintetizó el amiton a principios de 1952; aunque una fuente afirma que lo hizo en 1948, pero la patente del PPL no se solicitó hasta noviembre de 1952 y los detalles no se publicaron hasta 1955, cuando obtuvo esta protección, así como otras diversas patentes relacionadas con la preparación de la sustancia. Después, en 1957, se lanzó al mercado una forma de amiton como insecticida, conocida como tetram o amiton ICI. El tetram no tuvo éxito como insecticida, puesto que era demasiado tóxico para su utilización por seres humanos. Al igual que con el tabun, esto significaba que, potencialmente, era suficientemente tóxico para utilizarse contra seres humanos.

De vuelta en el Ministerio de Abastecimiento, en correspondencia con los nuevos códigos- $\mathrm{C}$ de 29 de abril de 1953, el primer compuesto para ser considerado por el nuevo sistema recibió el código C11. Esto era el amiton. En noviembre de 1953, el C11 y otro compuesto (al que se le dio después el código VE) se trataron en términos entusiastas por la CDAB: "Había aparecido, por tanto, una línea completamente nueva en el campo del gas nervioso, cuando se pensaba que estaba completamente circunscrito, y era inevitable que se arrojara una nueva luz sobre las relaciones entre estructura y actividad (structurelactivity relationships)"

En mayo de 1954, el Reino Unido había informado de los nuevos agentes a sus homólogos en los Estados Unidos (en Edgewood Arsenal) y en Canadá. El informe de 1954 de la CDAB señalaba que "se ha sintetizado una serie de nuevos compuestos de azufre y fósoforo y se ha dedicado mucho esfuerzo a preparar cada forma estructural en un estado puro". Y en noviembre de 1955 se hizo referencia a los agentes como los agentes $\mathrm{V}$ (venenosos) en documentos del Reino Unido. Dos años después, los científicos del CDEE informaron de que desde 1945 se habían preparado en el CDEE más de 200 compuestos organofosforados-anticolinesterásicos " $y$, probablemente, se han examinado más en Edgewood". En febrero de 1957, el Army Research and Development Command [Comando de Desarrollo e Investigación del Ejército] seleccionó el agente VX como el más venenoso.

Vale la pena señalar que, a pesar de la coincidencia entre los intereses de la industria de pesticidas comerciales y los intereses militares, sus agendas no estaban perfectamente sincronizadas. La meta de la industria pesticida era desarrollar sustancias químicas que fueran altamente tóxicas para los insectos, pero no para los mamíferos; mientras que el ejército buscaba sustancias químicas que fueran altamente tóxicas para los mamíferos. Esta discrepancia se puso de relieve en mayo de 1957, cuando el Ministerio de Abastecimiento envió una nueva ronda

\footnotetext{
${ }^{7}$ Prof. E. Jones TNA, WO195/12549, Ministerio de Abastecimiento, Chemical Defence Advisory
} Board. Acta 24 de la reunión de la Junta (5 de noviembre de 1953). 
de cartas, tanto directamente a compañías individuales como indirectamente a través de la Asociación Británica de Fábricas de Productos Químicos. Aunque, en general, las repuestas de la industria a las cartas ofrecían cooperación, algunas expresaban reservas. Por ejemplo, la respuesta de la compañía Fisons Pest Control S.A. decía: "En ocasiones encontramos compuestos que son excepcionalmente tóxicos para los mamíferos... pero, como comprenderán, esto es una señal para no trabajar más en ello y, a menudo, nuestra información en esta fase es escasa"8.

En cartas de las compañías Glaxo y Shell se expresaron opiniones similares.

La transferencia del amiton del PPL a Porton Down demuestra (demostrates) cómo el centro de defensa británico interactuaba con la industria química nacional (domestic chemical industry), para desarrollar una nueva familia de agentes nerviosos. Sin embargo, volviendo al problema conceptual más amplio de cómo pensar acerca del uso dual (dual-use), mantengo que no estaba preestablecido que el "amiton pesticida" se convirtiera en el "gas nervioso VG". A pesar de un cierto grado de superposición entre lo civil y lo militar, forjar las condiciones para la transferencia de Tecnología desde la industria química al sector militar requería un proceso activo de promoción. Expresado sin rodeos: el ICI no solo llamó a la puerta del CDEE con su nuevo descubrimiento, tuvo que hacer un uso dual. En este sentido, las autoridades de defensa británicas organizaron repetidas campañas de envío de cartas a la industria en busca de nuevos compuestos tóxicos, establecieron medidas de confidencialidad para proteger los secretos comerciales industriales y buscaron traducir las metas de la industria pesticida en las metas del laboratorio de guerra química. Aún así, estos esfuerzos no lograron generar una avalancha de nuevas líneas de investigación. En cambio, las empresas de productos químicos a las que se solicitó cooperación declararon repetidamente que, en el curso normal de los acontecimientos, no estarían interesadas en las mismas cosas que los científicos del Ejército.

Esta cuestión más conceptual —que las cosas no son simplemente de "usodual" (dual-use) o de "uso no-dual" (not dual-use), sino que tiene que hacerse un uso-dual - tiene implicaciones respecto a la forma en que enfocamos la parte práctica del control de armas químicas y biológicas. Para los que diseñan la actuación pública (policy-makers) contemporáneos, este aspecto del estudio de caso nos recuerda un punto fundamental acerca de la gobernanza (governance) de la Tecnología en la BWC y la CWC: su foco en el intento de regulación. Solo un foco más limitado sobre la Tecnología como tal da pie al "dilema" del uso dual, donde lo maligno y lo benigno se interpretan como inherentes al artefacto tecnológico (cfr. McCleish, 2007).

Por otra parte, los esfuerzos militares para obtener un pesticida y transformarlo en un arma química, junto con un entorno político que alienta la búsqueda

${ }^{8}$ TNA WO 188/2716, G. S. Hartley (Director of investigación) a E. E. Haddon, Director del Chemical Defence Research and Development, Ministerio de Abastecimiento, (4 de junio de 1957). 
de nuevos compuestos tóxicos, comporta la configuración activa de una red de artefactos y personas. De una manera significativa, estas actividades dejan huellas documentales que se pueden examinar. La intención (intent), que a menudo se considera como inefable y difícil de regular, aquí puede interpretarse como más conspicua y abierta al escrutinio regulador que los artefactos y los compuestos. Hay que reconocer que, en este caso, la búsqueda de la intención ha sido realizada por dos historiadores por placer, para reconstruir los eventos de modo más cuidadoso a partir de documentos, y más de medio siglo después de los acontecimientos. Así, claramente esta no es la misma tarea que atender, en cuanto gestores de la actuación pública, las situaciones inmediatas que incluyen Tecnologías de uso dual. Una vez señalado esto, el estudio de caso subraya el hecho de que la política requiere un amplio espectro de enfoque, más amplio que el mero artefacto tecnológico que considera, si vamos a hacer juicios razonables acerca de lo peligrosa que podría ser potencialmente una Tecnología de uso dual.

\section{Referencias bibliográficas}

Balmer, Brian (2001). Britain and Biological Warfare: Expert Advice and Science Policy, 1935-1965. Basingstoke: Palgrave.

BMA (1999). Biotechnology, Weapons and Humanity. Londres: British Medical Association.

Clunan, Anne, Lavoy, Peter R. y Martin, Susan B. (2008). Terrorism, War, or Disease? Unraveling the Use of Biological. Weapons, Stanford Security Studies, Stanford: Stanford University Press.

Cole, Leonard A. (2003). The Anthrax Letters: A Medical Detective Story. Washington DC: Joseph Henry Press.

Coleman, Kim (2005). A History of Chemical Warfare. Basingstoke: Palgrave.

Dando, Malcolm R. (1994). Biological Warfare in the 21 $1^{\text {st }}$ Century. Londres: Brasseys.

Dando, Malcolm R. (2002). Preventing Biological Warfare: The Failure of American Leadership (Global Issues). Basingstoke: Palgrave MacMillan.

Guillemin, Jeanne (1999). Anthrax: The Investigation of a Deadly Outbreak. Berkeley, CA: University of California Press.

Guillemin, Jeanne (2011). American Anthrax: Fear, Crime and the Investigation of the Nation's Deadliest Bioterror Attack. N. York: Henry Holt.

Harris, Robert y Paxman, Jeremy (1982). A Higher Form of Killing: The Secret Story of Gas and Germ Warfare. Londres: Chatto and Windus.

Kenyon, Ian (2000). Chemical weapons in the twentieth century: their use and their control. The CBW Conventions Bulletin, 48, 1-15. 
McLeish, Caitriona (2007). Reflecting on the Dual-Use Problem. En Brian Rappert y Caitriona McLeish (eds.), A Web of Prevention: Biological Weapons, Life Sciences and the Governance of Research (pp. 189-207). Londres: Earthscan.

McLeish, Caitriona y Balmer, Brian (2012). Development of the V-series nerve agents. En Jonathan B. Tucker (ed.), Innovation and Security: Preventing the Misuse of New Biological and Chemical Technologies (pp. 273-287). Cambridge, MA: The MIT Press.

Mayor, Adrienne (2003). Greek Fire, Poison Arrows and Scorpion Bombs: Biological and Chemical Warfare in the Ancient World. Londres: Duckworth.

Miller, Judith, Engelberg, Stephen y Broad, William (2001). Germs: Biological Weapons and America's Secret War. Londres: Simon and Schuster.

Reader, William J. (1975). Imperial Chemical Industries: A History. Vol. 2: The First Quarter-century, 1926-52. Oxford: Oxford University Press.

Selgelid, Michael J. (2007). A tale of two studies: Ethics, bioterrorism and the censorship of science. Hastings Center Report, 37(3), 35-43.

Strauss, Harlee y King, Jonathan (1986). The fallacy of defensive biological weapons programmes. En Erhard Geissler (ed.), Biological and Toxin Weapons Today (pp. 66-73). Oxford: Oxford University Press.

Tucker, Jonathan B. (1994). Dilemmas of a Dual-Use Technology: Toxins in Medicine and Warfare. Politics and the Life Sciences, 13(1), 51-62.

Tucker, Jonathan B. (2007). War of Nerves: Chemical Warfare from World War I to AlQaeda. N. York: Anchor Books.

Walker, John R. (2012). Britain and Disarmament: The UK and Nuclear, Biological and Chemical Weapons Arms Control and Programmes, 1956-1975. Farnham: Ashgate.

Wheelis, Mark, Rózsa, Lajos y Dando, Malcolm R. (eds.) (2006). Deadly Cultures: Biological Weapons Since 1945. Cambridge, MA: Harvard University Press. 